\title{
EDITORIAL
}

\section{Illustrating the wealth of urology}

\section{From}

launching the

competition to

finalizing the

exact colours for

the 12 issues, the

whole process

takes around

4 months
Every year, the clinical Nature Reviews journals launch their cover image competition and ask their readers to submit interesting and eye-catching images to grace the journal cover for the next 12 months. As you can imagine, an open request for people to send us interesting and eye-catching urological images can sometimes be fraught with difficulties, and our submissions are often the most interesting and eye-catching of all of those submitted to the clinical Reviews journals!

This year, as every year, we received many great submissions and so we decided to explain a little about what goes on behind the scenes and thank those who sent us depictions of their work by highlighting our shortlisted images in an Editorial. The four images that we shortlisted really illustrate the variety of subspecialties in urology - from neurourology to prostate morphology, penile transplant to biofilms - and offer fascinating insights into cutting-edge research.

Joanna Hannan and Elena Pak of East Carolina University sent us this fantastic image (FIG. 1a) of a co-culture of dissociated neurons plated on Schwann cells, both isolated from the major pelvic ganglia of a
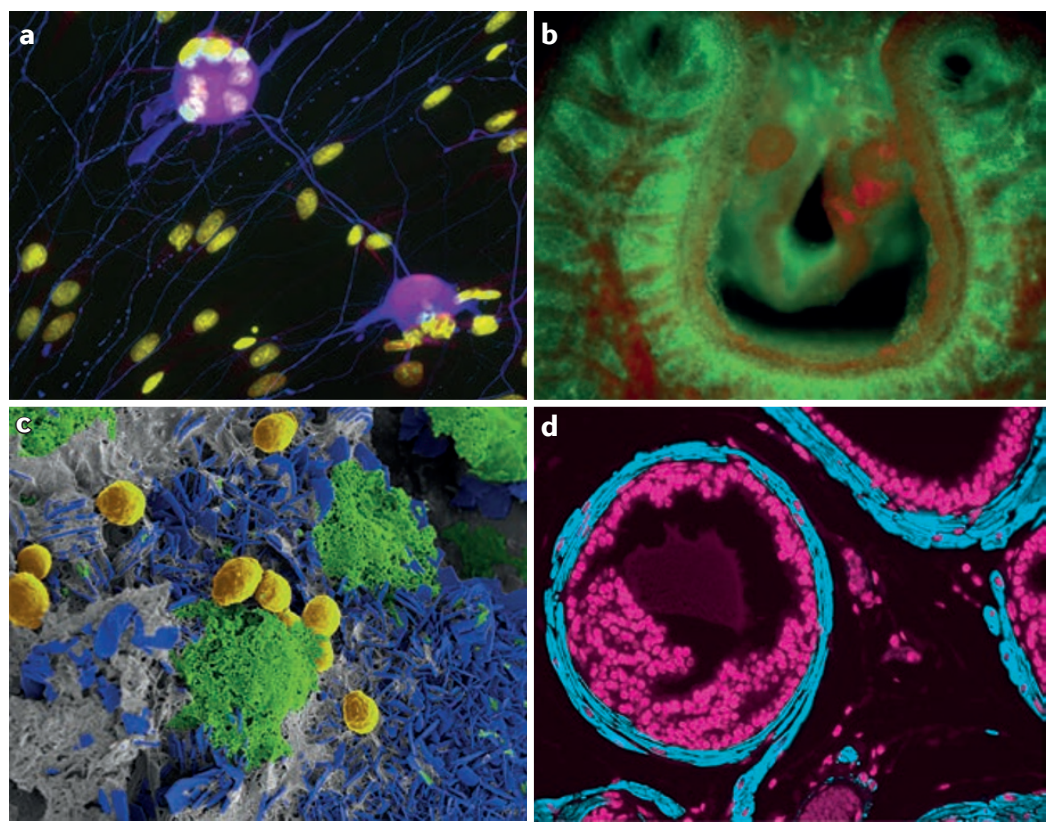

Figure 1 | 2016 cover competition shortlist. a | Dissociated neurons plated on Schwann cells. $\mathbf{b} \mid$ Rat penis undergoing apoptosis. $\mathbf{c}$ | Biofilm deposition on a ureteral stent. $\mathbf{d}$ | Prostate tissue. Part c is reproduced with permission from Zumstein, V., et al. Biofilm formation on ureteral stents - incidence, clinical impact, and prevention. Swiss Med Wkly 146, w14408 (2016).
Sprague-Dawley rat. The neurons are stained red with antibodies for beta III tubulin and the Schwann cells green with s100 antibody. They are interested in elucidating the role of Schwann cells in the regenerative process in neurons from the major pelvic ganglia in animal models of neurogenic erectile dysfunction.

Our second image (FIG. 1b) - the customary "dick pic" - came from Nikolai Sopko at Johns Hopkins, who has been working on the mechanisms and methods of penile transplantation in rat models. His live-image of a cultured rat penis cross-section undergoing apoptosis used SNARF-1 labelling to highlight all living cells and identified apoptotic cells containing activated caspase 3/7. "In this real-time ex vivo imaging of rat penile cross-section, red (SNARF) signal represents metabolically active tissues and green represents cells with activated caspase 3/7 undergoing apoptosis," explains Sopko. The image is taken at $40 \times$ magnification.

Valentin Zumstein and colleagues provided our third top image (FIG. 1 c), a scanning electron micrograph of biofilm deposition on a ureteral stent. "The biofilm contains hexagonal and amorphous crystals, a network of polymeric substances and attached microorganisms," Zumstein explains. "Prior to imaging, the sample was fixed with glutaraldehyde and formaldehyde in phosphate-buffered saline, followed by chemical dehydration and $\mathrm{Au} / \mathrm{Pd}$ sputtering."

However, there could only be one winner, and this year we chose this image of stained prostate tissue (FIG. 1 d) supplied by Sudeh Izadmehr at The Icahn School of Medicine at Mount Sinai, New York. The image was produced using fluorescence microscopy of mouse prostate glands stained for $\alpha$-smooth muscle actin and with DAPI staining of nuclei.

From launching the competition to finalizing the exact colours for the 12 issues, the whole process takes around 4 months. Once we have shortlisted a few images, our brilliant Production Editor, Hannah, painstakingly recolours them in a number of colourways, so we can then decide which one we would like to feature. When the final decision is made, she will then make a cover mock-up in up to 50 different colour schemes for the journal team to choose their final 12 to feature on the covers for the year.

We would like to extend our thanks to everyone who submitted images to this year's competition - we are so lucky to have such an interesting and varied field to cover in urology. We look forward to seeing what you send us next year! 